\title{
Uniqueness of fast travelling fronts in reaction-diffusion equations with delay
}

\author{
By Maitere Aguerrea, Sergei Trofimchuk* and Gabriel Valenzuela \\ Instituto de Matemática y Fisica, Universidad de Talca, Casilla 747, Talca, Chile
}

We consider positive travelling fronts $u(t, x)=\phi(\nu \cdot x+c t), \phi(-\infty)=0, \phi(\infty)=\kappa$, of the equation $u_{t}(t, x)=\Delta u(t, x)-u(t, x)+g(u(t-h, x)), x \in \mathbb{R}^{m}(*)$. It is assumed that $(*)$ has exactly two non-negative equilibria: $u_{1} \equiv 0$ and $u_{2} \equiv \kappa>0$. The birth function $g \in C^{2}(\mathbb{R}, \mathbb{R})$ may be non-monotone on $[0, \kappa]$. Hence, we are concerned with the so-called monostable case of the time-delayed reaction-diffusion equation. Our main result says that for every fixed and sufficiently large velocity $c$, the positive travelling front $\phi(\nu \cdot x+c t)$ is unique (modulo translations). Notice that $\phi$ can be non-monotone. To prove the uniqueness, we introduce a small parameter $\varepsilon=1 / c$ and realize the Lyapunov-Schmidt reduction in a scale of Banach spaces.

Keywords: Time-delayed reaction-diffusion equation; monostable case; uniqueness; travelling front; single species population model.

\section{Introduction and main result}

In this paper, we consider the time-delayed reaction-diffusion equation

$$
u_{t}(t, x)=\Delta u(t, x)-u(t, x)+g(u(t-h, x)), u(t, x) \geq 0, x \in \mathbb{R}^{m} .
$$

Eq. (1.1) and its non-local versions are used widely to model many physical, chemical, ecological and biological processes, see Faria et al. (2006) for more references. The nonlinearity $g$ is referred to in the ecology literature as the birth function, and we will suppose that $-x+g(x)$ is of the monostable type. Thus Eq. (1.1) has exactly two non-negative equilibria $u_{1} \equiv 0, u_{2} \equiv \kappa>0$. We say that the wave solution $u(x, t)=\phi(\nu \cdot x+c t),\|\nu\|=1$, of (1.1) is a wavefront (or a travelling front), if the profile function $\phi$ satisfies the boundary conditions $\phi(-\infty)=0$ and $\phi(+\infty)=\kappa$. After scaling, such a profile $\phi$ is a positive heteroclinic solution of the delay differential equation

$$
\varepsilon^{2} x^{\prime \prime}(t)-x^{\prime}(t)-x(t)+g(x(t-h))=0, \varepsilon:=1 / c>0, \quad t \in \mathbb{R} .
$$

Notice that $\phi$ may not be monotone. Since the biological interpretation of $u$ is the size of an adult population, we will consider only positive travelling fronts.

If we take $h=0$ in (1.1), we obtain a monostable reaction-diffusion equations without delay. The problem of existence of travelling fronts for this equation is quite well understood. In particular, for each such equation we can indicate a positive real number $c_{*}$ such that, for every $c \geq c_{*}$, it has exactly one travelling front $u(x, t)=\phi(\nu \cdot x+c t)$. Furthermore, Eq. (1.1) does not have any travelling front

*Author for correspondence (trofimch@inst-mat.utalca.cl) 
propagating at the velocity $c<c_{*}$. The profile $\phi$ is necessarily strictly increasing function. See, for example, Theorem 8.3 (ii), Theorem 8.7 and Theorem 2.39 in Gilding \& Kersner (2004).

However, the situation will change drastically if we take $h>0$. Actually, at the present moment, it seems that we are far from proving similar results concerning the existence, uniqueness and geometric properties of wavefronts for delayed equation (1.1). This despite that fact that the existence of travelling fronts in (1.1) was recently intensively studied for some specific subclasses of birth functions. E.g. see So, Wu \& Zou (2001), Wu \& Zou (2001), Faria et al. (2006), Ma (2007), Trofimchuk \& Trofimchuk (2008) and references wherein. Certainly, so called monotone case (when $g$ is monotone on $[0, \kappa]$ ) is that one for which the most information is available. But so far, even for equations with monotone birth functions very little is known about the number of wavefronts (modulo translation) for an arbitrary fixed $c \geq c_{*}$. In effect, there exist a very few theoretical studies devoted to the uniqueness problem for equations similar to (1.1). To the best of our knowledge, the uniqueness was established only for small delays in Ai (2007) and for a family of unimodal and piece-wise linear birth functions in Trofimchuk et al. (2007). The mentioned family is rather representative since 'asymmetric' tent maps mimic the main features of general unimodal birth functions. In fact, we believe that the uniqueness of positive wavefront can be proved for delayed equations with the unimodal birth function satisfying the following assumptions:

(H) The steady state $x_{1}(t) \equiv \kappa>0$ (respectively $\left.x_{2}(t) \equiv 0\right)$ of the equation

$$
x^{\prime}(t)=-x(t)+g(x(t-h))
$$

is exponentially stable and globally attractive (respectively hyperbolic).

(G) $g \in C^{1}\left(\mathbb{R}_{+}, \mathbb{R}_{+}\right), p:=g^{\prime}(0)>1$, and $g^{\prime \prime}(x)$ exists and is bounded near 0 . We suppose that $g$ has exactly two fixed points 0 and $\kappa>0$. Set $A=\sup \{a \in$ $\left.(0, \kappa / 2]: g^{\prime}(x)>0, x \in[0, a)\right\}$ and $\zeta_{2}=\max _{x \in[0, \kappa]} g(x)$, we assume that $g(x)>0$ for $x \in\left(0, \zeta_{2}\right]$. Then there exists a positive $\zeta_{1} \leq \min \left\{g\left(\zeta_{2}\right), A\right\}$ such that $g\left(\zeta_{1}\right)=\min _{s \in\left[\zeta_{1}, \zeta_{2}\right]} g(s)$. Notice that $g\left(\left[\zeta_{1}, \zeta_{2}\right]\right) \subseteq\left[\zeta_{1}, \zeta_{2}\right]$. Without restricting the generality, we can suppose that $\sup _{s \geq 0} g(s) \leq \zeta_{2}$.

In this paper, we follow the approach of Faria et al. (2006) to prove the uniqueness (up to translations) of positive wavefront for a given fast speed $c$. In the case of (1.1), this approach essentially relies on the fact that, in 'good' spaces and with suitable $g^{\prime}(0), g^{\prime}(\kappa)$, the linear operator $(\mathcal{L} x)(t)=x^{\prime}(t)+x(t)-g^{\prime}(\psi(t-h)) x(t-h)$ is a surjective Fredholm operator. Here $\psi$ is a heteroclinic solution of equation (1.2) considered with $\varepsilon=0$. In consequence, the Lyapunov-Schmidt reduction can be used to prove the existence of a smooth family of travelling fronts in some neighborhood of $\psi$. As it was shown in Faria \& Trofimchuk (2006) this family contains positive solutions as well. However, an important and natural question about the number of the positive wavefronts has not been answered in the past. We solve this problem in the present paper, establishing the following result:

Theorem 1.1. Assume $(\mathbf{H}),(\mathbf{G})$. Then there exists a unique (modulo translations) positive wavefront of Eq. (1.1) for each sufficiently large speed $c$. 
In order to apply Theorem 1.1 one needs to find sufficient conditions to ensure the global attractivity of the positive equilibrium of (1.3). Some results in this direction were found in Liz et al. (2005) for nonlinearities satisfying a generalized Yorke condition. In particular, Corollary 2.3 of the latter paper implies the following

Corollary 1.2. Assume $(\mathbf{H})$ and $(\mathbf{G})$, and that either $\Gamma:=g^{\prime}(\kappa) \in[0,1]$ or

$$
\Gamma<0 \quad \text { and } \quad e^{-h}>-\Gamma \ln \frac{\Gamma^{2}-\Gamma}{\Gamma^{2}+1} .
$$

Suppose also that $g \in C^{3}\left(\mathbb{R}_{+}, \mathbb{R}_{+}\right)$has only one critical point $x_{M}$ (maximum) and that the Schwarzian $(S g)(x)=g^{\prime \prime \prime}(x)\left(g^{\prime}(x)\right)^{-1}-(3 / 2)\left(g^{\prime \prime}(x)\left(g^{\prime}(x)\right)^{-1}\right)^{2}$ is negative for all $x>0, x \neq x_{M}$. Then the conclusion of Theorem 1.1 holds true.

Notice that Corollary 1.2 applies to both the Nicholson's blowflies equation and the Mackey-Glass equation with non-monotone nonlinearity.

The structure of this paper is as follows: the next section contains preliminary facts and fixes some notation. In the third section, following Faria et al. (2006), we realize the Lyapunov-Schmidt reduction in a scale of Banach spaces. Section 4 contains the core lemma of the paper. As an applications of this lemma, we obtain an alternative proof of the existence of positive wavefronts, see Theorem 4.2. Finally, in Section 5 we show that there exists exactly one wavefront for each fixed fast speed.

\section{Preliminaries}

This section contains several auxiliary results that will be needed later. Proofs of them (excepting Lemma 2.7) can be found in Liz et al. (2002) [Lemma 2.1], Trofimchuk et al. (2007) [Lemma 2.2, Faria \& Trofimchuk (2006) [Lemmas 2.3. 2.6.

Lemma 2.1. Assume (G). If $x \not \equiv 0$ is a non negative solution of Eq. (1.3), then

$$
\zeta_{1} \leq \liminf _{t \rightarrow+\infty} x(t) \leq \limsup _{t \rightarrow+\infty} x(t) \leq \zeta_{2} .
$$

Lemma 2.2. Assume $(\mathbf{G})$. Consider wavefront $u(x, t)=\phi(\nu \cdot x+c t),\|\nu\|=1$, to $E q$. (1.1). Then there exists a unique $\tau$ such that $\phi(\tau)=A, \phi^{\prime}(s)>0$ for all $s \leq \tau$.

Lemma 2.3. Suppose that $p>1$ and $h>0$. Then the characteristic equation

$$
z=-1+p \exp (-z h)
$$

has only one real root $0<\lambda<p-1$. Moreover, all roots $\lambda, \lambda_{j}, j=2,3, \ldots$ of (2.1) are simple and we can enumerate them in such a way that $\lambda>\Re \lambda_{2}=\Re \lambda_{3} \geq \ldots$

Everywhere in the sequel, $\lambda_{j}$ stands for a root of (2.1). Notice that we write $\lambda$ instead of $\lambda_{1}$.

Lemma 2.4. Assume (H), (G) and let $\lambda$ be as in Lemma 2.3. Then (1.3) has a unique (modulo translations) positive heteroclinic solution $\psi$. Moreover, $\psi\left(t-t_{0}\right)=$ $\exp (\lambda t)+O(\exp ((2 \lambda-\delta) t)), t \rightarrow-\infty$, for each $\delta>0$ and some $t_{0} \in \mathbb{R}$. 
Lemma 2.5. Let $\left\{\lambda_{\alpha}(\varepsilon), \alpha \in A\right\}$, where $\mathbb{N} \cup\{\infty\} \subset A$, denote the (countable) set of roots to the equation

$$
\varepsilon^{2} z^{2}-z-1+p \exp (-z h)=0 .
$$

If $p>1, h>0, \varepsilon \in(0,1 /(2 \sqrt{p-1}))$ then (2.2) has exactly two real roots $\lambda_{1}(\varepsilon), \lambda_{\infty}(\varepsilon)$ such that

$$
0<\lambda<\lambda_{1}(\varepsilon)<2(p-1)<\varepsilon^{-2}-2(p-1)<\lambda_{\infty}(\varepsilon)<\varepsilon^{-2}+1 .
$$

Moreover: (i) there exists an interval $\mathcal{O}=\mathcal{O}(p, h) \ni 0$ such that, for every $\varepsilon \in \mathcal{O}$, all roots $\lambda_{\alpha}(\varepsilon), \alpha \in A$ of (2.2) are simple and the functions $\lambda_{\alpha}: \mathcal{O} \rightarrow \mathbb{C}$ are continuous; (ii) we can enumerate $\lambda_{j}(\varepsilon), j \in \mathbb{N}$, in such a way that there exists $\lim _{\varepsilon \rightarrow 0+} \lambda_{j}(\varepsilon)=\lambda_{j}$ for each $j \in \mathbb{N}$, where $\lambda_{j} \in \mathbb{C}$ are the roots of (2.1), with $\lambda_{1}=\lambda$; (iii) for all sufficiently small $\varepsilon$, every vertical strip $\xi \leq \Re z \leq 2(p-1)$ contains only a finite set of $m(\xi)$ roots (if $\xi \notin\left\{\Re \lambda_{j}, j \in \mathbb{N}\right\}$, then $m(\xi)$ does not depend on $\varepsilon) \lambda_{1}(\varepsilon), \ldots, \lambda_{m(\xi)}(\varepsilon)$ to (2.2), while the half-plane $\Re z>2(p-1)$ contains only the root $\lambda_{\infty}(\varepsilon)$.

Assume $(\mathbf{H}),(\mathbf{G})$, and let $\psi$ be the positive heteroclinic solution from Lemma 2.4. For a fixed $\mu \geq 0$, we set $\#\left\{\lambda_{j}: \mu<\Re \lambda_{j}\right\}:=d(\mu)$ and $\|x\|^{+}=\sup _{\mathbb{R}_{+}}|x(s)|$, $\|x\|_{\mu}^{-}=\sup _{\mathbb{R}_{-}} e^{-\mu s}|x(s)|,|x|_{\mu}=\max \left\{\|x\|^{+},\|x\|_{\mu}^{-}\right\}$. Consider the Banach space

$$
C_{\mu}(\mathbb{R})=\left\{x \in C(\mathbb{R}, \mathbb{R}):\|x\|_{\mu}^{-}<\infty, x(-\infty)=0, \text { and } x(+\infty) \text { is finite }\right\},
$$

equipped with the norm $|x|_{\mu}$. We will need the operators $\mathcal{G}, \mathcal{I}, \mathcal{I}_{\varepsilon}, \mathcal{I}_{\varepsilon}^{+}, \mathcal{I}_{\varepsilon}^{-}, \mathcal{N}$ : $C_{\mu}(\mathbb{R}) \rightarrow C_{\mu}(\mathbb{R})$, where $(\mathcal{G} x)(t)=g(x(t))$ is the Nemitski operator, $\mathcal{I}=\mathcal{I}_{0}^{-}, \mathcal{I}_{0}^{+}=0$, $\mathcal{I}_{\varepsilon}=\sigma^{-1}(\varepsilon)\left(\mathcal{I}_{\varepsilon}^{+}+\mathcal{I}_{\varepsilon}^{-}\right), \sigma(\varepsilon):=\sqrt{1+4 \varepsilon^{2}}$, and

$$
\begin{gathered}
\left(\mathcal{I}_{\varepsilon}^{+} x\right)(t)=\int_{t}^{+\infty} e^{\frac{(1+\sigma(\varepsilon))(t-s)}{2 \varepsilon^{2}}} x(s-h) d s,\left(\mathcal{I}_{\varepsilon}^{-} x\right)(t)=\int_{-\infty}^{t} e^{\frac{-2(t-s)}{1+\sigma(\varepsilon)}} x(s-h) d s, \\
(\mathcal{N} x)(t)=\int_{-\infty}^{t} e^{-(t-s)} q(s) x(s-h) d s, q(s):=g^{\prime}(\psi(s-h)) .
\end{gathered}
$$

Since $g^{\prime}(x)=p+O(x), x \rightarrow 0$, and $\psi(t)=O(\exp (\lambda t)), t \rightarrow-\infty$, we obtain that

$$
q(t)=p+\epsilon(t), \epsilon(t)=O(\exp (\lambda t)), t \rightarrow-\infty ; \text { and } q(-\infty)=p>1, q(\infty)=g^{\prime}(\kappa) .
$$

Observe that $\mathcal{I}_{\varepsilon}^{ \pm}, \mathcal{N}$ are well defined: e.g. $(\mathcal{N} x)(+\infty)=g^{\prime}(\kappa) x(+\infty)$ and, for $t \leq h$,

$$
|(\mathcal{N} x)(t)| \leq \int_{-\infty}^{t} e^{-(t-s)}|q(s)|\|x\|_{\mu}^{-} e^{\mu(s-h)} d s \leq \frac{\|x\|_{\mu}^{-} \sup _{t \leq h}|q(t)|}{1+\mu} e^{\mu(t-h)}
$$

Lemma 2.6. Operator families $\mathcal{I}_{\varepsilon}^{ \pm}:(-1 / \sqrt{\mu}, 1 / \sqrt{\mu}) \rightarrow \mathcal{L}\left(C_{\mu}(\mathbb{R})\right), \mu \geq 0$, are continuous in the operator norm. In particular, $\mathcal{I}_{\varepsilon} \rightarrow \mathcal{I}$ as $\varepsilon \rightarrow 0$.

Lemma 2.7. If $(\mathbf{H})$ holds and $\mu \notin\left\{\Re \lambda_{j}\right\}, \mu \geq 0$, then $I-\mathcal{N}: C_{\mu}(\mathbb{R}) \rightarrow C_{\mu}(\mathbb{R})$ is a surjective Fredholm operator and $\operatorname{dim} \operatorname{Ker}(I-\mathcal{N})=d(\mu)$. 
Proof. First, we establish that $I-\mathcal{N}$ is an epimorphism. Take some $d \in C_{\mu}(\mathbb{R})$ and consider the following integral equation

$$
x(t)-\int_{-\infty}^{t} e^{-(t-s)} q(s) x(s-h) d s=d(t) .
$$

If we set $z(t)=x(t)-d(t)$, this equation is transformed into

$$
z(t)-\int_{-\infty}^{t} e^{-(t-s)} q(s)(z(s-h)+d(s-h)) d s=0 .
$$

Hence, in order to establish the surjectivity of $I-\mathcal{N}$, it suffices to prove the existence of $C_{\mu}(\mathbb{R})$-solution of the equation

$$
z^{\prime}(t)=-z(t)+q(t) z(t-h)+q(t) d(t-h) .
$$

First, notice that all solutions of (2.3) are bounded on the positive semi-axis $\mathbb{R}_{+}$due to the boundedness of $q(t) d(t-h)$ and the exponential stability of the homogeneous $\omega$-limit equation $z^{\prime}(t)=-z(t)+g^{\prime}(\kappa) z(t-h)$. Here we use the persistence of exponential stability under small bounded perturbations (e.g. see Section 5.2 in Chicone \& Latushkin (1999)) and the fact that $q(+\infty)=g^{\prime}(\kappa)$. Furthermore, since every solution $z$ of (2.3) satisfies $z^{\prime}(t)=-z(t)+g^{\prime}(\kappa) z(t-h)+g^{\prime}(\kappa) d(+\infty)+\epsilon(t)$ with $\epsilon(+\infty)=0$, we get $z(+\infty)=d(+\infty) g^{\prime}(\kappa)\left(1-g^{\prime}(\kappa)\right)^{-1}$. Next, by effecting the change of variables $z(t)=\exp (\mu t) y(t)$ to Eq. (2.3), we get a linear inhomogeneous equation of the form

$$
y^{\prime}(t)=-(1+\mu) y(t)+\left[p \exp (-\mu h)+\epsilon_{1}(t)\right] y(t-h)+\epsilon_{2, \mu}(t),
$$

where $\epsilon_{1}(-\infty)=\epsilon_{2,0}(-\infty)=0$ and $\epsilon_{2, \mu}(t)=O(1), \mu>0$, at $t=-\infty$. Since the $\alpha$-limit equation $y^{\prime}(t)=-(1+\mu) y(t)+p \exp (-\mu h) y(t-h), \mu \notin\left\{\Re \lambda_{j}\right\}$, to the homogeneous part of (2.4) is hyperbolic, due to the above mentioned persistence of the property of exponential dichotomy, we again conclude that Eq. (2.4) also has an exponential dichotomy on $\mathbb{R}_{-}$. Thus (2.4) has a solution $y_{\mu}^{*}$ which is bounded on $\mathbb{R}_{-}$(while $y_{0}^{*}(-\infty)=0$ ) so that $z^{*}(t)=\exp (\mu t) y_{\mu}^{*}(t)=O(\exp (\mu t)), t \rightarrow-\infty$, is a $C_{\mu}(\mathbb{R})$-solution of Eq. (2.3).

Next we prove that $\operatorname{dim} \operatorname{Ker}(I-\mathcal{N})=\#\left\{\lambda_{j}: \mu<\Re \lambda_{j}\right\}$. It is clear that $\phi_{j} \in \operatorname{Ker}(I-\mathcal{N})$ if and only if $\phi_{j}$ is a $C_{\mu}(\mathbb{R})$-solution of the equation

$$
\phi^{\prime}(t)=-\phi(t)+q(t) \phi(t-h) .
$$

We already have seen that every solution of (2.5) satisfies $\phi(+\infty)=0$, thus we only have to show that there exist solutions $\phi_{j}$ with $\left\|\phi_{j}\right\|_{\mu}<\infty$. In fact, we will prove that for each $\Re \lambda_{j}>\mu$ and $\delta \in\left(0, \min _{\Re \lambda_{j}>0, \lambda>\Re \lambda_{i}>0}\left\{\Re \lambda_{j}, \lambda-\Re \lambda_{i}\right\}\right)$ there is $\phi_{j}(t)=e^{\lambda_{j} t}+e^{\sigma t} v_{j}(t) \in \operatorname{Ker}(I-\mathcal{N})$, with $\sigma=\lambda+\delta, v_{j}(t)=O(1), t \rightarrow-\infty$. Set $q(t)=p+\epsilon(t)$, then $v_{j}(t)$ can be chosen as a bounded solution of the equation

$$
v^{\prime}(t)+(1+\sigma) v(t)-(p+\epsilon(t)) e^{-\sigma h} v(t-h)=e^{-\lambda_{j} h+\left(\lambda_{j}-\sigma\right) t} \epsilon(t) .
$$

Since $e^{-\lambda_{j} h+\left(\lambda_{j}-\sigma\right) t} \epsilon(t)=O\left(e^{\left(\Re \lambda_{j}-\delta\right) t}\right)$ at $-\infty$, we get the following $\alpha$-limit form of (2.6)

$$
v^{\prime}(t)+(1+\sigma) v(t)-p e^{-\sigma h} v(t-h)=0 .
$$


This autonomous equation is exponentially stable since its characteristic equation

$$
z+\lambda+\delta=-1+p e^{-(z+\lambda+\delta) h}
$$

has roots $z_{j}=\lambda_{j}-\lambda-\delta$ with $\Re z_{j}=\Re \lambda_{j}-\lambda-\delta<0$. Thus (2.6) has a unique solution $v_{j}$ bounded in $\mathbb{R}_{-}$. Is clear that $d(\mu)$ solutions $\left\{\phi_{j}\right\}$ are linearly independent, we claim that, in fact, system $\left\{\phi_{j}\right\}$ generates $\operatorname{Ker}(I-\mathcal{N})$. Indeed, suppose for an instance that $\phi \in \operatorname{Ker}(I-\mathcal{N})-<\phi_{j}>$.

As $\phi$ solves the equation

$$
x^{\prime}(t)=-x(t)+p x(t-h)+O(\exp ((\lambda+\mu) t)), t \rightarrow-\infty,
$$

we get (e.g. p. 28 in Mallet-Paret (1999))

$$
\phi(t)=z(t)+O(\exp ((\lambda+\mu-\delta) t)), t \rightarrow-\infty,
$$

where $z(t)$ is the eigensolution corresponding to the eigenvalues $\zeta$ with $\mu \leq \Re \zeta<$ $\lambda+\mu$. In this way,

$$
\phi(t)=C \exp (\lambda t)+\sum_{j=2}^{d(\mu)} C_{j} \exp \left(\lambda_{j} t\right)+O(\exp ((\lambda+\mu-\delta) t)), t \rightarrow-\infty .
$$

Now take

$$
w(t)=C\left(\exp (\lambda t)+\exp (\sigma t) v_{1}(t)\right)+\sum_{j=2}^{d(\mu)} C_{j}\left(\exp \left(\lambda_{j} t\right)+\exp (\sigma t) v_{j}(t)\right) \in<\phi_{j}>.
$$

Since $\exp (\sigma t) v_{j}(t)=O(\exp (\lambda+\delta) t), t \rightarrow-\infty$, we can write

$$
w(t)=C \exp (\lambda t)+\sum_{j=2}^{d(\mu)} C_{j} \exp \left(\lambda_{j} t\right)+O(\exp ((\lambda+\delta) t)), t \rightarrow-\infty .
$$

Thus $\Delta(t):=\phi(t)-w(t)$ satisfies $\Delta(t)=O(\exp (\lambda-\delta) t), t \rightarrow-\infty$, and solves

$$
x^{\prime}(t)=-x(t)+p x(t-h)+O(\exp ((2 \lambda-\delta) t)), t \rightarrow-\infty .
$$

Applying Proposition 7.1 from Mallet-Paret (1999) we conclude that

$$
\Delta(t)=z(t)+O(\exp ((2 \lambda-\delta-\delta / 2) t)), t \rightarrow-\infty,
$$

where $z(t)$ is the eigensolution corresponding to the eigenvalues $\zeta$ such that $\lambda-\delta \leq$ $\Re \zeta<2 \lambda-\delta$ and in consequence $z(t)=C_{1} e^{\lambda t}$, for some $C_{1}$. Hence,

$$
\phi(t)=w(t)+\Delta(t)=C^{\prime} \exp (\lambda t)+\sum_{j=2}^{d(\mu)} C_{j} \exp \left(\lambda_{j} t\right)+O(\exp ((\lambda+\delta) t)), t \rightarrow-\infty
$$

for small $\delta>0$. The latter formula improves (2.7), and if we take

$$
w_{1}(t)=C^{\prime}\left(\exp (\lambda t)+\exp (\sigma t) v_{1}(t)\right)+\sum_{j=2}^{d(\mu)} C_{j}\left(\exp \left(\lambda_{j} t\right)+\exp (\sigma t) v_{j}(t)\right) \in<\phi_{j}>
$$


then $\Delta_{1}(t)=\phi(t)-w_{1}(t)=O(\exp (\lambda+\delta) t), t \rightarrow-\infty$. Since $\Delta_{1}(t)$ satisfies

$$
x^{\prime}(t)=-x(t)+p x(t-h)+O(\exp ((2 \lambda+\delta) t)), t \rightarrow-\infty,
$$

we can proceed as before to get $\Delta_{1}(t)=z_{1}(t)+O(\exp (2 \lambda+\delta-\delta / 2) t), t \rightarrow-\infty$, where $z_{1}(t)$ is the eigensolution corresponding to the eigenvalues $\zeta$ such that $\lambda+\delta \leq$ $\zeta<2 \lambda+\delta$. Thus $z_{1}(t)=0$ and $\Delta_{1}(t)=O(\exp (2 \lambda+\delta-\delta / 2) t), t \rightarrow-\infty$. Iterating this procedure (and subtracting $\delta / 2^{k}$ from the exponent $2 \lambda+\delta$ on the step $k$ ), we can conclude that $\Delta_{1}(t)=O(\exp (k \lambda t)), t \rightarrow-\infty, k \geq 2$. This means that $\Delta$ is a small solution of (2.5). However, Eq. (2.5) cannot have solutions with superexponential decay at $-\infty$ (e.g see p. 9 in Faria \& Trofimchuk (2006)) and thus $\Delta(t)=0$. This implies that $\phi \in<\phi_{j}>$, a contradiction.

Throughout the rest of the paper, we will suppose that the $C^{1}$-smooth function $g$ is defined and bounded on the whole real axis $\mathbb{R}$. This assumption does not restrict the generality of our framework, since it suffices to take any smooth and bounded extension on $\mathbb{R}_{-}$of the nonlinearity $g$ described in $(\mathbf{G})$. Notice that, since there exists finite $g^{\prime}(0)$, we have $g(x)=x \gamma(x)$ for a bounded $\gamma \in C(\mathbb{R})$. Set $\gamma_{0}=\sup _{t \in \mathbb{R}}|\gamma(x)|$. As it can be easily checked, $|\mathcal{G} x|_{\mu} \leq \gamma_{0}|x|_{\mu}$ so that actually $\mathcal{G}$ is well-defined. Furthermore, we have the following lemma:

Lemma 2.8. Assume that $g \in C^{1}(\mathbb{R})$. Then $\mathcal{G}$ is Fréchet continuously differentiable on $C_{\mu}(\mathbb{R})$ with differential $\mathcal{G}^{\prime}\left(x_{0}\right): y(\cdot) \rightarrow g^{\prime}\left(x_{0}(\cdot)\right) y(\cdot)$.

Proof. We have that $\left|\mathcal{G}^{\prime}(x) u\right|_{\mu}=\left|g^{\prime}(x(\cdot)) u(\cdot)\right|_{\mu} \leq \sup _{t \in \mathbb{R}}\left|g^{\prime}(x(t))\right||u|_{\mu}$. By the Taylor formula, $g(v)-g\left(v_{0}\right)-g^{\prime}\left(v_{0}\right)\left(v-v_{0}\right)=\left(g^{\prime}(\theta)-g^{\prime}\left(v_{0}\right)\right)\left(v-v_{0}\right), \quad \theta \in\left[v, v_{0}\right]$. Fix some $x_{0} \in C_{\mu}(\mathbb{R})$. Since functions in $C_{\mu}(\mathbb{R})$ are bounded and $g^{\prime}$ is uniformly continuous on bounded sets of $\mathbb{R}$, for any given $\delta>0$ there is $\sigma>0$ such that for $\left|x-x_{0}\right|_{\mu}<\sigma$ we have that $\left|\mathcal{G} x-\mathcal{G} x_{0}-g^{\prime}\left(x_{0}(\cdot)\right)\left(x-x_{0}\right)\right|_{\mu} \leq \delta\left|x-x_{0}\right|_{\mu}$ and $\left\|\mathcal{G}^{\prime}(x)-\mathcal{G}^{\prime}\left(x_{0}\right)\right\|_{\mathcal{L}\left(C_{\mu}(\mathbb{R})\right)}<\delta$.

\section{Lyapunov-Shmidt reduction}

Being a bounded solution of Eq. (1.2), each travelling wave should satisfy

$$
x(t)=\frac{1}{\sigma(\varepsilon)}\left(\int_{-\infty}^{t} e^{\frac{-2(t-s)}{1+\sigma(\varepsilon)}} g(x(s-h)) d s+\int_{t}^{+\infty} e^{\frac{(1+\sigma(\varepsilon))(t-s)}{2 \varepsilon^{2}}} g(x(s-h)) d s\right),
$$

For $C_{\mu}(\mathbb{R})$-solutions, this equation takes the form $x=\left(\mathcal{I}_{\varepsilon} \circ \mathcal{G}\right) x$.

Theorem 3.1. Assume $(\mathbf{H}),(\mathbf{G})$. Let $\psi$ be the positive heteroclinic from Lemma 2.4 Then for every $\mu \neq \Re \lambda_{j}, \mu \in[0, \lambda)$, there are open balls $\mathcal{E}_{\mu}=\left(-\varepsilon_{\mu}, \varepsilon_{\mu}\right)$, $\mathcal{V}_{\mu} \subset \mathbb{R}^{d(\mu)}$, and continuous family of heteroclinics $\psi_{\varepsilon, v}: \mathcal{E}_{\mu} \times \mathcal{V}_{\mu} \rightarrow C_{\mu}(\mathbb{R})$ of Eq. (1.2) such that $\psi_{0,0}=\psi$. For each $\tilde{\varepsilon} \in \mathcal{E}_{\mu}$, the subset $\left\{\psi_{\tilde{\varepsilon}, v}: v \in \mathcal{V}_{\mu}\right\} \subset C_{\mu}(\mathbb{R})$ is $C^{1}$-manifold of dimension $d(\mu)$. Moreover, there exists a $C_{\mu}(\mathbb{R})$-neighborhood $\mathcal{U}$ of $\psi$ and $\varepsilon_{1}>0$ such that every solution $\psi_{\varepsilon} \in \mathcal{U},|\varepsilon|<\varepsilon_{1}$, of Eq. (1.2) satisfies $\psi_{\varepsilon}=\psi_{\varepsilon, v}$ for some $v \in \mathcal{V}_{\mu}$. Finally, given a closed subinterval $\mathcal{S} \subset[0, \lambda) \backslash\left\{\Re \lambda_{j}\right\}$, we can choose open sets $\mathcal{E}_{\mu}, \mathcal{V}_{\mu}$ to be constant on $\mathcal{S}$. 
Proof. Set $R_{\mu}=(-1 / \sqrt{\mu}, 1 / \sqrt{\mu})$ and then define $F: R_{\mu} \times C_{\mu}(\mathbb{R}) \rightarrow C_{\mu}(\mathbb{R})$ by $F(\varepsilon, \phi)=\psi+\phi-\left(\mathcal{I}_{\varepsilon} \circ \mathcal{G}\right)(\psi+\phi)$. We have that $F(0,0)=0$. Furthermore, Lemmas 2.6 and 2.8 imply that $F \in C\left(R_{\mu} \times C_{\mu}(\mathbb{R}), C_{\mu}(\mathbb{R})\right)$ and $F_{\phi}(\varepsilon, \phi)$ is continuous in a neighborhood of $(0,0)$. Set

$$
L:=F_{\phi}(0,0)=I-\mathcal{N}, V:=\operatorname{Ker} L, r(\varepsilon, \phi):=F(\varepsilon, \phi)-L \phi .
$$

Then $r_{\phi}(0,0)=F_{\phi}(0,0)-L=0$. By Lemma 2.7, we have that $\operatorname{dim} V<\infty$ and that $L$ is surjective. Thus $V$ has a topological complement $W$ in $C_{\mu}(\mathbb{R})$ so that $C_{\mu}(\mathbb{R})=V \oplus W$ and any $\phi \in C_{\mu}(\mathbb{R})$ can be written in the form $\phi=v+w, v \in V$ and $w \in W$. Recalling that $L v=0$ we get $F(\varepsilon, \phi)=L w+r(\varepsilon, v+w)$. This suggests the following definition:

$$
\Phi(\varepsilon, v, w):=\left.L\right|_{W} w+r(\varepsilon, v+w)
$$

where $\Phi_{w}(0,0,0)=\left.L\right|_{W}$ is the restriction of $L$ to $W$. Is clear that $\Phi \in C\left(R_{\mu} \times V \times\right.$ $\left.W, C_{\mu}(\mathbb{R})\right)$ and $\Phi_{w}(\varepsilon, v, w)=\left.L\right|_{W}+r_{\phi}(\varepsilon, v+w)$ is continuous in a neighborhood of $(0,0,0)$. Since $\left.L\right|_{W}: W \rightarrow C_{\mu}(\mathbb{R})$ is bijective we have that $\left(\left.L\right|_{W}\right)^{-1}$ is continuous from $C_{\mu}(\mathbb{R})$ to $W$. As a consequence, we can apply the Implicit Function Theorem (e.g. see Theorem 2.3(i) in Ambrosetti \& Prodi (1993)) to

$$
\Phi(\varepsilon, v, w)=\left.L\right|_{W} w+r(\varepsilon, v+w)=0, \quad \Phi(0,0,0)=0 .
$$

In this way, we find neighborhoods of $0, \mathcal{E}_{\mu} \subset R_{\mu}, \mathcal{V}_{\mu} \subset V$ and $\mathcal{W}_{\mu} \subset W$ and a continuous map $\gamma \in C_{v}^{1}\left(\mathcal{E}_{\mu} \times \mathcal{V}_{\mu}, \mathcal{W}_{\mu}\right)$, such that $\Phi(\varepsilon, v, \gamma(\varepsilon, v))=0$ for all $(\varepsilon, v) \in \mathcal{E}_{\mu} \times \mathcal{V}_{\mu}$. Moreover, without restricting the generality, we can suppose that $\Phi(\varepsilon, v, w)=0$ with $(\varepsilon, v, w) \in \mathcal{E}_{\mu} \times \mathcal{V}_{\mu} \times \mathcal{W}_{\mu}$ implies $w=\gamma(\varepsilon, v)$ (e.g. see Theorem 2.3(ii) in Ambrosetti \& Prodi (1993)).

Hence, the continuous family $\psi_{\varepsilon, v}=\psi+v+\gamma(\varepsilon, v): \mathcal{E}_{\mu} \times \mathcal{V}_{\mu} \rightarrow C_{\mu}(\mathbb{R})$ contains all solutions of Eq. (1.2) from small neighborhoods of $\psi$, with $\psi_{0,0}=\psi$. Since $\gamma_{v}(0,0)=0$ and $\gamma_{v}(\varepsilon, v)$ is continuous for each fixed $\varepsilon \in \mathcal{E}_{\mu}$, we conclude that $\left\{\psi_{\varepsilon, v}: v \in \mathcal{V}_{\mu}\right\} \subset C_{\mu}(\mathbb{R})$ is $C^{1}$-smooth manifold of dimension $d(\mu)$. Notice that (3.1) implies that $g\left(\psi_{\varepsilon, v}(+\infty)\right)=\psi_{\varepsilon, v}(+\infty)$. Thus $\psi_{\varepsilon, v}(+\infty)=\psi_{0,0}(+\infty)=\kappa$, so that $\left\{\psi_{\varepsilon, v}\right\}$ are heteroclinic solutions of (1.2).

Finally, the last conclusion of the theorem follows from the simple observations that (a) the sets $\mathcal{E}_{\mu}, \mathcal{V}_{\mu}, \mathcal{W}_{\mu}$ are non-increasing in $\mu$ and (b) the function $d(t)$ is piece-wise constant, with discontinuities at $\left\{\Re \lambda_{j}\right\} \cap[0, \lambda)$.

\section{Asymptotic formulae}

Throughout this section, we denote by $\beta, \gamma, \eta, b, C, C_{j}, C_{*}, \ldots$ some positive constants that are independent of the parameters $\varepsilon \in \Lambda_{j}:=\left(-\varepsilon_{j}, \varepsilon_{j}\right), v \in \Omega$, where $1>\varepsilon_{0}>\varepsilon_{1}>\cdots>\varepsilon_{*}>0$, and $\Omega \subset \mathbb{R}^{q}$. We also assume that $h>0, p>1$.

Lemma 4.1. Let continuous $x_{\varepsilon, v}(\cdot), f_{\varepsilon, v}(\cdot): \Lambda_{0} \times \Omega \times \mathbb{R} \rightarrow \mathbb{R}$ satisfy

$$
\varepsilon^{2} x^{\prime \prime}(t)+x^{\prime}(t)-x(t)+p x(t+h)=f_{\varepsilon, v}(t), \quad t \in \mathbb{R} .
$$

Suppose further that $\sup _{t \leq 0}\left[\left|x_{\varepsilon, v}(t)\right|+\left|f_{\varepsilon, v}(t)\right|\right] \leq C, \quad\left|x_{\varepsilon, v}(t)\right| \leq C e^{-\gamma t}, t \geq 0$, and that $\left|f_{\varepsilon, v}(t)\right| \leq C e^{-b t}, t \geq 0,(\varepsilon, v) \in \Lambda_{0} \times \Omega$. Then, given $\sigma \in(0, b)$, it holds

$$
x_{\varepsilon, v}(t)=z_{\varepsilon, v}(t)+w_{\varepsilon, v}(t), t \in \mathbb{R},
$$


where, with some continuous and bounded $B_{j}:\left(-\varepsilon_{*}, \varepsilon_{*}\right) \times \Omega \rightarrow \mathbb{C}$,

$$
z_{\varepsilon, v}(t)=\sum_{\gamma \leq \Re \lambda_{j}(\varepsilon)<b-\sigma} B_{j}(\varepsilon, v) e^{-\lambda_{j}(\varepsilon) t}
$$

is a finite sum of eigensolutions of (4.1) associated to the roots $\lambda_{j}(\varepsilon) \in\{\gamma \leq$ $\left.\Re \lambda_{j}(\varepsilon)<b-\sigma\right\}$ of (2.2) and $\left|w_{\varepsilon, v}(t)\right| \leq C_{*} e^{-(b-\sigma) t}, t \geq 0,(\varepsilon, v) \in\left(-\varepsilon_{*}, \varepsilon_{*}\right) \times \Omega$.

Proof. Applying the Laplace transform $\mathcal{L}$ to equation (4.1), we obtain

$$
\chi(z, \varepsilon) \tilde{x}_{\varepsilon, v}(z)=\tilde{f}_{\varepsilon, v}(z)+r_{\varepsilon, v}(z),
$$

where $\chi(z, \varepsilon)=\varepsilon^{2} z^{2}+z-1+p \exp (z h), \tilde{x}_{\varepsilon, v}=\mathcal{L}\left\{x_{\varepsilon, v}\right\}, \tilde{f}_{\varepsilon, v}=\mathcal{L}\left\{f_{\varepsilon, v}\right\}$, and

$$
r_{\varepsilon, v}(z)=\varepsilon^{2}\left(x_{\varepsilon, v}^{\prime}(0)+z x_{\varepsilon, v}(0)\right)+x_{\varepsilon, v}(0)+p e^{z h} \int_{0}^{h} e^{-z u} x_{\varepsilon, v}(u) d u .
$$

Since $x_{\varepsilon, v} e^{\gamma t}$ is bounded, $\tilde{x}_{\varepsilon, v}$ is holomorphic in the open half-plane $\{\Re z>-\gamma\}$. Similarly, $\tilde{f}_{\varepsilon, v}$ is holomorphic in $\{\Re z>-b\}$. Since $r_{\varepsilon, v}$ is entire, the function

$$
H_{\varepsilon, v}(z):=\left(\tilde{f}_{\varepsilon, v}(z)+r_{\varepsilon, v}(z)\right) / \chi(z, \varepsilon)
$$

is meromorphic in $\Re z>-b$, with only finitely many poles there.

Step I. We claim that there are $\sigma^{\prime} \in(0, \sigma), \varepsilon_{1}>0$, such that $\left|H_{\varepsilon, v}(z)\right| \leq C_{1} /|z|$, if $\Re z=-b+\sigma^{\prime},(\varepsilon, v) \in \Lambda_{1} \times \Omega$. Indeed, take $\sigma^{\prime} \in(0, \sigma)$ such that the line $\Re z=-b+\sigma^{\prime}$ does not contain any eigenvalue $-\lambda_{j}(\varepsilon), \varepsilon \in \bar{\Lambda}_{1}$, and $1-b+\sigma^{\prime} \neq 0$. We have

$$
\begin{aligned}
& \left|\tilde{f}_{\varepsilon, v}(z)\right| \leq \int_{0}^{+\infty} e^{-\Re z t}\left|f_{\varepsilon, v}(t)\right| d t \leq C \int_{0}^{+\infty} e^{-\Re z t} e^{-b t} d t \leq \frac{C}{\sigma^{\prime}}, \Re z \geq-b+\sigma^{\prime} \\
& \left|r_{\varepsilon, v}(z)\right| \leq \varepsilon^{2}\left(\left|x_{\varepsilon, v}^{\prime}(0)\right|+|z|\left|x_{\varepsilon, v}(0)\right|\right)+\left|x_{\varepsilon, v}(0)\right|+p e^{\Re z h} \int_{0}^{h} e^{-\Re z u}\left|x_{\varepsilon, v}(u)\right| d u .
\end{aligned}
$$

As a bounded solution of (4.1), $x_{\varepsilon, v}$ should satisfy, for all $t \in \mathbb{R}$,

$$
x_{\varepsilon, v}(t)=\frac{1}{\sqrt{1+4 \varepsilon^{2}}}\left(\int_{-\infty}^{t} e^{\bar{\lambda}(t-s)} G_{\varepsilon, v}(s) d s+\int_{t}^{+\infty} e^{\bar{\mu}(t-s)} G_{\varepsilon, v}(s) d s\right),
$$

where $\bar{\lambda}<0<\bar{\mu}$ are the roots of $\varepsilon^{2} z^{2}+z-1=0$ and $G_{\varepsilon, v}(t):=p x_{\varepsilon, v}(t+h)-f_{\varepsilon, v}(t)$. Differentiating (4.2), we obtain

$$
x_{\varepsilon, v}^{\prime}(t)=\frac{1}{\sqrt{1+4 \varepsilon^{2}}}\left(\bar{\lambda} \int_{-\infty}^{t} e^{\bar{\lambda}(t-s)} G_{\varepsilon, v}(s) d s+\bar{\mu} \int_{t}^{+\infty} e^{\bar{\mu}(t-s)} G_{\varepsilon, v}(s) d s\right),
$$

so that

$$
\begin{aligned}
\left|x_{\varepsilon, v}^{\prime}(0)\right| \leq & \frac{\bar{\mu}}{\sqrt{1+4 \varepsilon^{2}}} \int_{0}^{+\infty} e^{-\bar{\mu} s}\left|G_{\varepsilon, v}(s)\right| d s+\frac{|\bar{\lambda}|}{\sqrt{1+4 \varepsilon^{2}}} \int_{-\infty}^{0} e^{-\bar{\lambda} s}\left|G_{\varepsilon, v}(s)\right| d s \leq \\
& (p+1) C\left(\int_{0}^{+\infty} \bar{\mu} e^{-\bar{\mu} s} d s+|\bar{\lambda}| \int_{-\infty}^{0} e^{-\bar{\lambda} s} d s\right)=2 C(p+1) .
\end{aligned}
$$


Fix $k>-b+\sigma^{\prime}$ and consider the vertical strip $\Sigma_{k}:=\left\{-b+\sigma^{\prime} \leq \Re z \leq k\right\}$, then

$$
p e^{\Re z h} \int_{0}^{h}\left|e^{-z u} x_{\varepsilon, v}(u)\right| d u \leq C p e^{k h} \int_{0}^{h} e^{b u} d u:=C_{3}, z \in \Sigma_{k},
$$

so that $\left|r_{\varepsilon, v}(z)\right| \leq C_{4}\left(1+\varepsilon^{2}|z|\right), z \in \Sigma_{k}$.

Set $b(z)=-1+p e^{z h}$, then $|b(z)| \leq 1+p e^{k h}:=\beta, z \in \Sigma_{k}$, and

$$
|z|\left|H_{\varepsilon, v}(z)\right| \leq \frac{C_{5}\left(|z|+\varepsilon^{2}|z|^{2}\right)}{\left|\varepsilon^{2} z^{2}+z+b(z)\right|}, \quad z \in \Sigma_{k}
$$

Now, set $y_{0}=\eta \beta$ for some $\eta>2$ satisfying $\eta^{2} \geq 2 \beta^{-1} \sqrt{\eta^{2} \beta^{2}+b^{2}}$ and $\eta \beta>b-\sigma^{\prime}$. For all $z$ such that $\Re z=-b+\sigma^{\prime}$, and $|\Im z| \geq y_{0}$, we have

$$
\left|\varepsilon z^{2}+z\right|=|z|\left|\varepsilon^{2} z+1\right| \geq y_{0}\left|\varepsilon^{2} z+1\right| \geq \frac{y_{0}^{2}}{\sqrt{y_{0}^{2}+\left(b-\sigma^{\prime}\right)^{2}}} \geq 2 \beta .
$$

Thus $\left|\varepsilon^{2} z^{2}+z+b(z)\right| \geq\left|\varepsilon^{2} z^{2}+z\right|-|b(z)| \geq\left|\varepsilon^{2} z^{2}+z\right|-\beta \geq\left|\varepsilon^{2} z^{2}+z\right| / 2$, so that

$$
\frac{\left(|z|+\varepsilon^{2}|z|^{2}\right)}{\left|\varepsilon^{2} z^{2}+z+b(z)\right|} \leq 2 \frac{1+\varepsilon^{2}|z|}{\left|\varepsilon^{2} z+1\right|} \leq \eta+\sup _{\Re z=-b+\sigma^{\prime}} \frac{2\left|\varepsilon^{2} z\right|}{\left|\varepsilon^{2} z+1\right|} \leq 2 \eta
$$

for all $|\Im z| \geq y_{0}, \Re z=-b+\sigma^{\prime}$ and $\varepsilon \in \Lambda_{1}$.

Finally, for all $(z, \varepsilon) \in\left\{z: \Re z=-b+\sigma^{\prime},|\Im z| \leq y_{0}\right\} \times \bar{\Lambda}_{1}$, we have that

$$
\frac{|z|+\varepsilon|z|^{2}}{\left|\varepsilon z^{2}+z+b(z)\right|} \leq C_{6}
$$

Combining this inequality with (4.4), (4.5), we prove the main assertion of Step I. Step II. Taking $k>0$, in virtue of (4.4) we can use the inversion formula

$$
x_{\varepsilon, v}(t)=\frac{1}{2 \pi i} \int_{k-\infty i}^{k+\infty i} e^{z t} \tilde{x}_{\varepsilon, v}(z) d z=\frac{1}{2 \pi i} \int_{k-\infty i}^{k+\infty i} e^{z t} H_{\varepsilon, v}(z) d z, t \geq 0 .
$$

By Lemma 2.5. $H_{\varepsilon, v}(z)$ has only finitely many poles in the strip $-b<\Re z \leq-\gamma$. Also, $H_{\varepsilon, v}(z) \rightarrow 0$ uniformly in the strip $-b+\sigma^{\prime} \leq \Re z \leq k$, as $|\Im z| \rightarrow \infty$, and $H_{\varepsilon, v}\left(-b+\sigma^{\prime}+i \cdot\right) \in L_{2}$. Thus, we may shift the path of integration in (4.6) to the left, to the line $\Re z=-b+\sigma^{\prime}$, and obtain $x_{\varepsilon, v}(t)=z_{\varepsilon, v}(t)+w_{\varepsilon, v}(t)$, where

$$
z_{\varepsilon, v}(t)=\sum_{\gamma \leq \Re \lambda_{j}(\varepsilon)<b-\sigma^{\prime}} \operatorname{Res}_{-\lambda_{j}(\varepsilon)} e^{z t} H_{\varepsilon, v}(z), w_{\varepsilon, v}(t)=\frac{1}{2 \pi i} \int_{-b+\sigma^{\prime}-\infty \cdot i}^{-b+\sigma^{\prime}+\infty \cdot i} e^{z t} H_{\varepsilon, v}(z) d z .
$$

By Lemma 2.5, the roots of equation $\chi(z, \varepsilon)=0$ are simple for all small $\varepsilon$. Hence

$z_{\varepsilon, v}(t)=\sum_{\gamma \leq \Re \lambda_{j}(\varepsilon)<b-\sigma^{\prime}} e^{-\lambda_{j}(\varepsilon) t} B_{j}(\varepsilon, v)$, with $B_{j}(\varepsilon, v)=\frac{\tilde{f}_{\varepsilon, v}\left(-\lambda_{j}(\varepsilon)\right)+r_{\varepsilon, v}\left(-\lambda_{j}(\varepsilon)\right)}{\chi^{\prime}\left(-\lambda_{j}(\varepsilon), \varepsilon\right)}$.

It is easy to check that $B_{j}(\varepsilon, v)$ is continuous on its domain of definition (observe here that the continuity of $x_{\varepsilon, v}^{\prime}(0)$ follows from (4.3) $)$. Take $j$ such that $-b+\sigma^{\prime}<$ 
$-\Re \lambda_{j}(\varepsilon) \leq-\gamma$, then $\left|r_{\varepsilon, v}\left(-\lambda_{j}(\varepsilon)\right)\right| \leq C_{4}\left(\varepsilon^{2}\left|\lambda_{j}(\varepsilon)\right|+1\right) \leq C_{4}\left(\max _{j, \varepsilon}\left|\lambda_{j}(\varepsilon)\right|+1\right):=C_{7}$.

In addition, if $\varepsilon \rightarrow 0$ then

$$
0<\left|\chi^{\prime}\left(-\lambda_{j}(\varepsilon), \varepsilon\right)\right|=\left|-2 \varepsilon^{2} \lambda_{j}(\varepsilon)+1+p h e^{-\lambda_{j}(\varepsilon) h}\right| \rightarrow\left|1+p h e^{-\lambda_{j} h}\right| \neq 0 .
$$

Hence, $\left|B_{j}(\varepsilon, v)\right| \leq \frac{\left|\tilde{f}_{\varepsilon, v}\left(-\lambda_{j}(\varepsilon)\right)\right|+\left|r_{\varepsilon, v}\left(-\lambda_{j}(\varepsilon)\right)\right|}{\left|\chi^{\prime}\left(-\lambda_{j}(\varepsilon), \varepsilon\right)\right|} \leq \frac{C / \sigma^{\prime}+C_{7}}{\min _{j, \varepsilon}\left|\chi^{\prime}\left(-\lambda_{j}(\varepsilon), \varepsilon\right)\right|} \leq C_{8}$

if $\varepsilon \in \Lambda_{2}$, for some small $\varepsilon_{2}>0$ and $v \in \Omega$.

Step III. Consider $u_{\varepsilon, v}(t)=e^{\left(b-\sigma^{\prime}\right) t} w_{\varepsilon, v}(t)$ and $v_{\varepsilon, v}(t)=e^{(b-\sigma) t} w_{\varepsilon, v}(t)$. We have

$u_{\varepsilon, v}(t)=\frac{1}{2 \pi i} \int_{-b+\sigma^{\prime}-\infty \cdot i}^{-b+\sigma^{\prime}+\infty \cdot i} e^{\left(s+b-\sigma^{\prime}\right) t} H_{\varepsilon, v}(s) d s=\frac{1}{2 \pi} \int_{-\infty}^{+\infty} e^{i \xi t} H_{\varepsilon, v}\left(-b+\sigma^{\prime}+i \xi\right) d \xi$.

By Plancherel theorem,

$$
\left\|u_{\varepsilon, v}\right\|_{2}=\frac{1}{2 \pi}\left\|H_{\varepsilon, v}\left(-b+\sigma^{\prime}+i \cdot\right)\right\|_{2} \leq \frac{C_{1}}{2 \sqrt{\pi\left(b-\sigma^{\prime}\right)}} .
$$

Hence, $v_{\varepsilon, v}(t)=e^{-\left(\sigma-\sigma^{\prime}\right) t} u_{\varepsilon, v}(t)$ is integrable on $[0,+\infty)$, and by the CauchySchwarz inequality

$$
\left\|v_{\varepsilon, v}\right\|_{1} \leq \frac{\left\|u_{\varepsilon, v}\right\|_{2}}{\sqrt{2\left(\sigma-\sigma^{\prime}\right)}} \leq \frac{C_{1}}{2 \sqrt{2 \pi\left(b-\sigma^{\prime}\right)\left(\sigma-\sigma^{\prime}\right)}} .
$$

Step $I V$. We claim that there exist real numbers $C_{9}>0$ and $\varepsilon_{3}>0$ such that $\overline{\left|w_{\varepsilon, v}(t)\right|} \leq C_{9} e^{-(b-\sigma) t}, t \geq 0$, for all $(\varepsilon, v) \in \Lambda_{3} \times \Omega$. In order to prove this, it suffices to show that $v_{\varepsilon, v}$ is uniformly bounded for small $\varepsilon \in \Lambda_{3}$. Since

$$
\varepsilon^{2} w_{\varepsilon, v}^{\prime \prime}(t)+w_{\varepsilon, v}^{\prime}(t)-w_{\varepsilon, v}(t)+p w_{\varepsilon, v}(t+h)=f_{\varepsilon, v}(t), \quad t \in \mathbb{R},
$$

we find that $v_{\varepsilon, v}(t)=e^{(b-\sigma) t} w_{\varepsilon, v}(t)$ satisfies

$$
\varepsilon^{2} v_{\varepsilon, v}^{\prime \prime}(t)+\left(1-2 \varepsilon^{2}(b-\sigma)\right) v_{\varepsilon, v}^{\prime}(t)=P_{\varepsilon, v}(t),
$$

where $\alpha=1-2 \varepsilon^{2}(b-\sigma)>0$ and $P_{\varepsilon, v} \in L_{1}[0,+\infty)$ is defined by

$$
P_{\varepsilon, v}(t)=e^{(b-\sigma) t} f_{\varepsilon, v}(t)+\left(1+(b-\sigma)-\varepsilon^{2}(b-\sigma)^{2}\right) v_{\varepsilon, v}(t)-p e^{-(b-\sigma) h} v_{\varepsilon, v}(t+h) .
$$

The variation of constants formula yields

$$
v_{\varepsilon, v}^{\prime}(t)=e^{-\frac{\alpha}{\varepsilon^{2}} t}\left(v_{\varepsilon, v}^{\prime}(0)+\frac{1}{\varepsilon^{2}} \int_{0}^{t} e^{\frac{\alpha}{\varepsilon^{2}} s} P_{\varepsilon, v}(s) d s\right), \varepsilon \neq 0 .
$$

A direct integration of (4.7) gives

$$
v_{\varepsilon, v}(t)=v_{\varepsilon, v}(0)+\frac{\varepsilon^{2}}{\alpha} v_{\varepsilon, v}^{\prime}(0)\left(1-e^{-\frac{\alpha}{\varepsilon^{2}} t}\right)+\frac{1}{\varepsilon^{2}} \int_{0}^{t} \int_{0}^{u} e^{\frac{\alpha}{\varepsilon^{2}}(s-u)} P_{\varepsilon, v}(s) d s d u .
$$


After changing the order of integration in the iterated integral, we get

$\frac{1}{\varepsilon^{2}}\left|\int_{0}^{t} \int_{s}^{t} e^{\frac{\alpha}{\varepsilon^{2}}(s-u)} P_{\varepsilon, v}(s) d u d s\right|=\frac{1}{\alpha}\left|\int_{0}^{t} P_{\varepsilon, v}(s)\left(1-e^{\frac{\alpha}{\varepsilon^{2}}(s-t)}\right) d s\right| \leq \frac{1}{\alpha} \int_{0}^{t}\left|P_{\varepsilon, v}(s)\right| d s$.

Additionally, recalling Step II, we find that $\left|v_{\varepsilon, v}^{\prime}(0)\right| \leq(b-\sigma)\left|w_{\varepsilon, v}(0)\right|+\left|w_{\varepsilon, v}^{\prime}(0)\right| \leq$

$$
\leq(b-\sigma)\left(\left|x_{\varepsilon, v}(0)\right|+\left|z_{\varepsilon, v}(0)\right|\right)+\left|x_{\varepsilon, v}^{\prime}(0)\right|+\left|z_{\varepsilon, v}^{\prime}(0)\right|<C_{10} .
$$

As a consequence, for all small $\varepsilon$ and $v \in \Omega$, we have that

$$
\left|v_{\varepsilon, v}(t)\right| \leq\left|v_{\varepsilon, v}(0)\right|+\frac{\varepsilon^{2}}{\alpha} C_{10}\left(1+e^{-\frac{\alpha}{\varepsilon^{2}} t}\right)+\frac{1}{\alpha} \int_{0}^{+\infty}\left|P_{\varepsilon, v}(s)\right| d s \leq C_{11}, t \geq 0 .
$$

Finally, since $w_{\varepsilon, v}(t)=v_{\varepsilon, v}(t) e^{-(b-\sigma) t}$, Lemma 4.1 is proved.

Theorem 4.2. In Theorem 3.1, take $\mu=\lambda-\delta$, with small $\delta>0$. Assume that $\psi$ is the positive heteroclinic of (1.3) normalized by $\psi(t)=\exp (\lambda t)+O(\exp ((2 \lambda-\delta) t))$, $t \rightarrow-\infty$. Then we can choose a neighborhood $\mathcal{U} \subset C_{\mu}(\mathbb{R})$ of $\psi$ and a neighborhood $\mathcal{E}_{\mu}^{*} \times \mathcal{V}_{\mu}^{*}$ of $0 \in \mathbb{R}^{2}$ in such a way that $\psi_{\varepsilon, v} \in \mathcal{U},(\varepsilon, v) \in \mathcal{E}_{\mu}^{*} \times \mathcal{V}_{\mu}^{*}$, is positive and unique in $\mathcal{U}$ (up to translations in $t$ ) for every fixed $\varepsilon$. Moreover, $\psi_{\varepsilon, v}\left(t-t_{0}\right)=$ $\exp \left(\lambda_{1}(\varepsilon) t\right)+O(\exp (1.99 \mu t))$ at $t \rightarrow-\infty$ for some $t_{0}=t_{0}(\varepsilon, v) \in \mathbb{R}$.

Proof. First, we take $\mathcal{V}_{\mu}, \mathcal{E}_{\mu} \subset\left(-\varepsilon_{1}, \varepsilon_{1}\right), \mathcal{U}$ as in Theorem 3.1. It follows from Lemma 2.5 and Theorem 3.1 that $\mathcal{V}_{\mu} \subset \mathbb{R}$ and that we can choose positive $\delta$ and $\mathcal{E}_{\mu}$ such that $\Re \lambda_{j}(\varepsilon)<\mu<\lambda<\lambda_{1}(\varepsilon)<1.99 \mu<\lambda_{\infty}(\varepsilon)$ for all $\varepsilon \in \mathcal{E}_{\mu}$. If we set $y_{\varepsilon, v}(t)=\psi_{\varepsilon, v}(-t)$, then $y_{\varepsilon, v}$ satisfies (4.1) where

$$
\left|f_{\varepsilon, v}(t)\right|=\left|g\left(y_{\varepsilon, v}(t+h)\right)-g^{\prime}(0) y_{\varepsilon, v}(t+h)\right| \leq C_{1} e^{-2 \mu t}, t \geq-h .
$$

Lemma 4.1 assures that there are $\mathcal{V}_{\mu}^{\prime} \subset \mathcal{V}_{\mu}, \mathcal{E}_{\mu}^{\prime} \subset \mathcal{E}_{\mu}$ such that

$$
y_{\varepsilon, v}(t)=B(\varepsilon, v) e^{-\lambda_{1}(\varepsilon) t}+w_{\varepsilon, v}(t),(\varepsilon, v) \in \mathcal{E}_{\mu}^{\prime} \times \mathcal{V}_{\mu}^{\prime} .
$$

Here $B: \mathcal{E}_{\mu}^{\prime} \times \mathcal{V}_{\mu}^{\prime} \rightarrow \mathbb{R}_{+}, B(0,0)=1$, is continuous and $\left|w_{\varepsilon, v}(t)\right| \leq C_{*} e^{-1.99 \mu t}$, $t \geq 0$, for some $C_{*}>0$.

Hence, there are $\mathcal{E}_{\mu}^{\prime \prime} \times \mathcal{V}_{\mu}^{\prime \prime}$ and $T>0$ (independent of $\varepsilon, v$ ) such that $y_{\varepsilon, v}(t)>$ $0.5 e^{-\lambda_{1}(\varepsilon) t}, t>T$, for all $(\varepsilon, v) \in \mathcal{E}_{\mu}^{\prime \prime} \times \mathcal{V}_{\mu}^{\prime \prime}$. On the other side, $\lim _{(\varepsilon, v) \rightarrow 0} y_{\varepsilon, v}(t)=$ $\psi(-t)$ uniformly on $\mathbb{R}$. In consequence, since $\psi$ is bounded from below by a positive constant on $[-T, \infty)$, we conclude that $y_{\varepsilon, v}$ is positive on $\mathbb{R}$, if $(\varepsilon, v)$ belongs to sufficiently small neighborhood $\mathcal{E}_{\mu}^{*} \times \mathcal{V}_{\mu}^{*} \subset \mathcal{E}_{\mu}^{\prime \prime} \times \mathcal{V}_{\mu}^{\prime \prime}$ of the origin. Without the loss of the generality, we can assume additionally that $\psi_{\varepsilon, v} \in \mathcal{U}$ for all $(\varepsilon, v) \in \mathcal{E}_{\mu}^{*} \times \mathcal{V}_{\mu}^{*}$.

Next, for every fixed $\varepsilon \in \mathcal{E}_{\mu}^{*}$, the subset $\mathfrak{F}=\left\{\psi_{\varepsilon, v}: v \in \mathcal{V}_{\mu}\right\} \subset C_{\mu}(\mathbb{R})$ is homeomorphic to $\mathcal{V}_{\mu}$. On the other hand, for every $n>0$, the collection $\mathfrak{P}_{n}=$ $\left\{\psi_{\varepsilon, 0}(t-s), s \in(-n, n)\right\}$ of positive heteroclinics is a continuous 1-manifold in $C_{\mu}(\mathbb{R})$. Since $\psi_{\varepsilon, 0} \in \mathfrak{F} \cap \mathfrak{P}_{n}$ we obtain that $\left\{\psi_{\varepsilon, v}: v \in \mathcal{V}_{\mu}^{*}\right\} \subset \mathfrak{P}_{\infty}$. In consequence, $\psi_{\varepsilon, v}(t)$ is unique in $\mathcal{U}$ (up to shifts in $t$ ) for every fixed small $\varepsilon$.

Theorem 4.3. Set $\mathcal{P}=\left\{(\varepsilon, v) \in \mathcal{E}_{0} \times \mathcal{V}_{0}: \psi_{\varepsilon, v}(t)>0, t \in \mathbb{R}\right\}$, where $\mathcal{E}_{0}$, $\mathcal{V}_{0}$ are as in Theorem 3.1. Then there exist a neighborhood $\mathcal{E}^{*} \times \mathcal{V}^{*} \subset \mathcal{E}_{0} \times \mathcal{V}_{0}$ of 0 and $C>0$ such that, for all $(\varepsilon, v) \in \mathcal{P}^{*}:=\mathcal{P} \cap\left(\mathcal{E}^{*} \times \mathcal{V}^{*}\right)$, we have that

$$
\psi_{\varepsilon, v}(t)=B(\varepsilon, v) e^{\lambda_{1}(\varepsilon) t}+w_{\varepsilon, v}(t),
$$

where $\left|w_{\varepsilon, v}(t)\right| \leq C e^{1.99 \lambda t}, t \leq 0$, and $B: \mathcal{E}^{*} \times \mathcal{V}^{*} \rightarrow(0, \infty)$ is continuous. 
Proof. Let $\mathcal{E}^{\prime} \subset \mathcal{E}_{0}$ be such that $\lambda_{\infty}(\varepsilon)>3 \lambda$, for all $\varepsilon \in \mathcal{E}^{\prime}$. The last assertion of Theorem 3.1 implies that, for some $\gamma>0, C_{1}>0$,

$$
\sup _{t \geq 0}\left|\psi_{\varepsilon, v}(t)\right| \leq C_{1},\left|\psi_{\varepsilon, v}(t)\right| \leq C_{1} e^{\gamma t}, t \leq 0 .
$$

If we set $y_{\varepsilon, v}(t)=\psi_{\varepsilon, v}(-t)$, then $y_{\varepsilon, v}$ satisfies (4.1) where

$$
\left|f_{\varepsilon, v}(t)\right|=\left|g\left(y_{\varepsilon, v}(t+h)\right)-g^{\prime}(0) y_{\varepsilon, v}(t+h)\right| \leq C_{2} e^{-2 \gamma t}, t \geq-h .
$$

Set $\Gamma=\sup \left\{\gamma>0\right.$ such that (4.9) holds for all $\left.(\varepsilon, v) \in \mathcal{P} \cap\left(\mathcal{E}^{\prime} \times \mathcal{V}_{0}\right)\right\}$. Applying Lemma 4.1, we get

$$
y_{\varepsilon, v}(t)=\sum_{0<\lambda_{j}(\varepsilon)<2 \Gamma} B_{j}(\varepsilon, v) e^{-\lambda_{j}(\varepsilon) t}+\tilde{w}_{\varepsilon, v}(t),
$$

where $B_{j}: \mathcal{E}^{\prime \prime} \times \mathcal{V}_{0} \rightarrow \mathbb{C}$ are continuous and $\left|\tilde{w}_{\varepsilon, v}(t)\right| \leq C_{3} e^{-1.99 \Gamma t}, t \geq 0,(\varepsilon, v) \in$ $\mathcal{P} \cap\left(\mathcal{E}^{\prime \prime} \times \mathcal{V}_{0}\right)$, for some $C_{3}>0$ and open $\mathcal{E}^{\prime \prime} \subset \mathcal{E}^{\prime}$. Since $\Gamma>0$ is finite and $y_{\varepsilon, v}(t)>0$, we obtain

$$
\sum_{0<\lambda_{j}(\varepsilon)<2 \Gamma} B_{j}(\varepsilon, v) e^{-\lambda_{j}(\varepsilon) t}=B(\varepsilon, v) e^{-\lambda_{1}(\varepsilon) t}
$$

so that $\Gamma \geq \lambda$, see Lemma 2.5 Next, due to Lemma 2.4 it holds that $B(0,0)>0$. Hence, $\Gamma=\lambda$.

Corollary 4.4. Given $\delta \in(0, \lambda)$ and $\left(\varepsilon_{j}, v_{j}\right) \in \mathcal{P}^{*}, j=0,1, \ldots$, the convergence

$$
\psi_{\varepsilon_{j}, v_{j}} \stackrel{C_{0}(\mathbb{R})}{\longrightarrow} \psi_{\varepsilon_{0}, v_{0}} \quad \text { implies } \quad \psi_{\varepsilon_{j}, v_{j}} \stackrel{C_{\lambda-\delta}(\mathbb{R})}{\longrightarrow} \psi_{\varepsilon_{0}, v_{0}} .
$$

Proof. By the contrary, suppose that there are a sequence $\left\{\psi_{\varepsilon_{j}, v_{j}},\left(\varepsilon_{j}, v_{j}\right) \in \mathcal{P}^{*}\right\}_{j \geq 0}$ and $\eta>0$ such that

$$
\lim _{j}\left|\psi_{\varepsilon_{j}, v_{j}}-\psi_{\varepsilon_{0}, v_{0}}\right|_{0}=0,\left|\psi_{\varepsilon_{j}, v_{j}}-\psi_{\varepsilon_{0}, v_{0}}\right|_{\lambda-\delta}>\eta, j=1,2, \ldots
$$

It follows from (4.8) that there exist $C>0$ and $T<0$ such that

$$
\psi_{\varepsilon_{j}, v_{j}}(t) e^{-(\lambda-\delta) t} \leq C e^{\delta t}<\eta / 4, j=0,1,2, \ldots, t \leq T .
$$

Thus

$$
\sup _{s \leq T}\left[e^{-(\lambda-\delta) s}\left|\psi_{\varepsilon_{j}, v_{j}}(s)-\psi_{\varepsilon_{0}, v_{0}}(s)\right|\right] \leq \eta / 2, j=1,2, \ldots
$$

Next, since $\psi_{\varepsilon_{j}, v_{j}}(t) \rightarrow \psi_{\varepsilon_{0}, v_{0}}(t)$ uniformly on $\mathbb{R}$, we can find $j_{*}$ such that

$$
\sup _{s \in[T, 0]}\left[e^{-(\lambda-\delta) s}\left|\psi_{\varepsilon_{j}, v_{j}}(s)-\psi_{\varepsilon_{0}, v_{0}}(s)\right|\right] \leq \frac{\eta}{2}, \sup _{s \geq 0}\left|\psi_{\varepsilon_{j}, v_{j}}(s)-\psi_{\varepsilon_{0}, v_{0}}(s)\right| \leq \frac{\eta}{2}, j \geq j_{*} .
$$

But all this means that $\left|\psi_{\varepsilon_{j}, v_{j}}-\psi_{\varepsilon_{0}, v_{0}}\right|_{\lambda-\delta} \leq \eta / 2$ for all $j \geq j_{*}$, a contradiction. 


\section{Proof of Theorem 1.1}

Everywhere below, all positive wavefronts $\phi$ will be normalized by the conditions $\phi(0)=\zeta_{1} / 2$ and $\phi(s)<\zeta_{1} / 2, s<0$, with $\zeta_{1}$ defined in $(\mathbf{G})$. Let $\psi, \psi(0)=\zeta_{1} / 2$, $\psi(s)<\zeta_{1} / 2, s<0$, be the positive heteroclinic of (1.3) given in Lemma 2.4. By Theorem 4.2, there exists a neighborhood $\left(-\varepsilon_{0}, \varepsilon_{0}\right) \times \mathcal{U} \subset \mathbb{R} \times C_{\lambda-\delta}(\mathbb{R})$ of $(0, \psi)$ such that for every fixed $\varepsilon \in\left(-\varepsilon_{0}, \varepsilon_{0}\right)$ there is a unique normalized positive wavefront $\psi_{\varepsilon} \in \mathcal{U}$. We claim that, if $\varepsilon$ is sufficiently small, then this $\psi_{\varepsilon}$ will be the unique normalized positive wavefront of Eq. (1.2). Indeed, let us suppose, for instance, that we can find a sequence $\varepsilon_{j} \rightarrow 0$ and normalized positive wavefronts $\phi_{\varepsilon_{j}} \neq \psi_{\varepsilon_{j}}$.

Lemma 5.1. Assume $(\mathbf{H})$ and $(\mathbf{G})$. Then $\phi_{\varepsilon_{j}} \rightarrow \psi$ uniformly on $\mathbb{R}$.

Proof. First, we prove the uniform convergence $\phi_{\varepsilon_{j}} \rightarrow \psi$ on compact subsets of $\mathbb{R}$. Since $g$ is a bounded function, we obtain from (3.1) that

$$
\left|\phi_{\varepsilon_{j}}^{\prime}(t)\right|+\left|\phi_{\varepsilon_{j}}(t)\right| \leq \frac{\max _{x \geq 0} g(x)}{\varepsilon^{2}(\mu-\lambda)}+\max _{x \geq 0} g(x) \leq 2 \zeta_{2}, j \in \mathbb{N} .
$$

Hence, by the Ascoli-Arzelà theorem combined with the diagonal method, $\left\{\phi_{\varepsilon_{j}}\right\}$ is precompact in $C(\mathbb{R}, \mathbb{R})$. Thus, every $\left\{\phi_{\varepsilon_{j_{k}}}\right\}$ has a subsequence converging in $C(\mathbb{R}, \mathbb{R})$ to some continuous positive bounded function $\varphi(s)$ such that $\varphi^{\prime}(s) \geq 0, s \leq 0$, and $\varphi(0)=\zeta_{1} / 2$. Making use of the Lebesgue's dominated convergence theorem, we deduce from Eq. (3.1) that

$$
\varphi(t)=\int_{-\infty}^{t} e^{-(t-s)} g(\varphi(s-h)) d s
$$

Therefore $\varphi$ is a positive bounded solution of Eq. (1.3) and since the equilibrium $\kappa$ of Eq. (1.3) is globally attractive, it holds that $\varphi(+\infty)=\kappa$. On the other hand, since $\varphi(-\infty) \leq \varphi(0)=\zeta_{1} / 2$, we have that $\varphi(-\infty)=0$. Hence, due to Lemma 2.4. we obtain that $\varphi(t)=\psi(t), t \in \mathbb{R}$. Next, if $\varphi_{\varepsilon_{n}} \not \rightarrow \psi$ uniformly on $\mathbb{R}$ then there exist a subsequence $\left\{\varphi_{\varepsilon_{j_{n}}}\right\} \subset\left\{\varphi_{\varepsilon_{j}}\right\}$ (for short, we will write again $\left\{\varphi_{\varepsilon_{j}}\right\}$ instead of $\left\{\varphi_{\varepsilon_{j_{n}}}\right\}$ ), a sequence $\left\{S_{j}\right\}$ and positive numbers $T, \delta<\kappa / 6$ such that

$$
\left|\psi\left(S_{j}\right)-\varphi_{\varepsilon_{j}}\left(S_{j}\right)\right|=2 \delta,|\psi(t)|<0.25 \delta, t \leq-T,|\psi(t)-\kappa|<0.25 \delta, t \geq T .
$$

Since $\varphi_{\varepsilon_{n}}$ converges uniformly on $[-2 T, 2 T]$ to $\psi$, and $\varphi_{\varepsilon_{n}}, \psi$ are monotone increasing on $(-\infty, 0]$, we can suppose that $\left|\psi(t)-\varphi_{\varepsilon_{n}}(t)\right|<\delta$ for all $t \in(-\infty, 2 T]$ and $n \geq n_{0}$. In this way, $S_{j} \rightarrow+\infty$ and we can suppose that

$$
\left|\psi(t)-\varphi_{\varepsilon_{j}}(t)\right|<2 \delta, t \in\left(-\infty, S_{j}\right) .
$$

Consider the sequence $y_{j}(t)=\varphi_{\varepsilon_{j}}\left(t+S_{j}\right)$ of heteroclinics to Eq. (1.2). We have that $\left|y_{j}(0)-\kappa\right|>1.5 \delta$ and $\left|y_{j}(t)-\kappa\right|<3 \delta$ when $t \in\left(T-S_{j}, 0\right)$. Arguing as above, we find that $\left\{y_{j}\right\}$ contains a subsequence converging, on compact subsets of $\mathbb{R}$, to some solution $y_{*}(t)$ of (1.3) satisfying $\left|y_{*}(0)-\kappa\right| \geq 1.5 \delta$ and $\left|y_{*}(t)-\kappa\right| \leq 3 \delta<\frac{\kappa}{2}$ for all $t<0$. Lemma 2.1 implies that $\inf _{\mathbb{R}} y_{*}(t)>0$. Since $y_{*}(0) \neq \kappa$, we have established the existence of a non-constant positive bounded and separated from 0 solution to (1.3). This contradicts to the global attractivity of $\kappa$. 
Corollary 5.2. $\phi_{\varepsilon_{j}} \rightarrow \psi$ in $C_{\lambda-\delta}(\mathbb{R})$.

Proof. Since $\phi_{\varepsilon_{j}} \rightarrow \psi$ in $C_{0}(\mathbb{R})$, we have that $\phi_{\varepsilon_{j}}=\psi_{\varepsilon_{j}, v_{j}}$ for some $v_{j} \in \mathcal{V}_{0}$. Now we can apply Corollary 4.4 to find that $\phi_{\varepsilon_{j}} \rightarrow \psi$ in $C_{\lambda-\delta}(\mathbb{R})$.

Lastly, Theorem 4.2 and Corollary 5.2 implies that $\phi_{\varepsilon_{j}}=\psi_{\varepsilon_{j}}$, a contradiction which completes the proof of Theorem 1.1 .

\section{Acknowledgments}

The authors thank Teresa Faria for useful discussions. S. Trofimchuk was partially supported by CONICYT (Chile) through PBCT program ACT-05 and by the University of Talca, program "Reticulados y Ecuaciones". S. Trofimchuk and G. Valenzuela were supported by FONDECYT (Chile) project 1071053.

\section{References}

Ai S. 2007 Traveling wave fronts for generalized Fisher equations with spatio-temporal delays, J. Differ. Equations, 232, 104-133.

Ambrosetti A. \& Prodi G. 1993 A Primer of Nonlinear Analysis. Cambridge: Cambridge University Press.

Chicone C. \& Latushkin Y. 1999 Evolution Semigroups in Dynamical Systems and Differential Equations. Providence, RI: American Mathematical Society.

Faria T., Huang W. \& Wu J. 2006 Traveling waves for delayed reaction-diffusion equations with non-local response, Proc. R. Soc. A 462, 229-261.

Faria T. \& Trofimchuk S. 2006, Non-monotone travelling waves in a single species reactiondiffusion equation with delay, J. Differ. Equations, 228, 357-376.

Gilding B. \& Kersner R. 2004, Travelling Waves in Nonlinear Diffusion-Convection Reaction. Basel: Birkhauser Verlag.

Gourley S. A., So J. \& Wu J. 2004 Non-locality of reaction-diffusion equations induced by delay: biological modeling and nonlinear dynamics, J. Math. Sciences, 124, 5119-5153.

Liz E., Martínez C. \& Trofimchuk S. 2002, Attractivity properties of infinite delay MackeyGlass type equations, Differ. and Integral Equations, 15, 875-896.

Liz E., Pinto M., Tkachenko V. \& Trofimchuk S., 2005 A global stability criterion for a family of delayed population models, Quart. Appl. Math., 63, 56-70.

Ma S. 2007 Traveling waves for non-local delayed diffusion equations via auxiliary equations, J. Differ. Equations, 237, 259-277.

Mallet-Paret J. 1999 The Fredholm alternative for functional differential equations of mixed type, J. Dyn. Differ. Equations, 11, 1-48.

So J., Wu J. \& Zou X. 2001 A reaction-diffusion model for a single species with age structure. I. Travelling wavefronts on unbounded domains, Proc. R. Soc. A 457, 18411853.

Trofimchuk E., Tkachenko V. \& Trofimchuk S. 2007 Slowly oscillating wave solutions of a single species reaction-diffusion equation with delay, Oberwolfach Preprints Series: OWP 2007-12, http://www.mfo.de/publications/owp/2007/OWP2007_12.pdf

Trofimchuk E. \& Trofimchuk S. 2008 Admissible wavefront speeds for a single species reaction-diffusion equation with delay, Discrete Contin. Dyn. Systems A, 20, 407-423.

Wu J. \& Zou X. 2001, Traveling wave fronts of reaction-diffusion systems with delay, $J$. Dyn. Differ. Equations, 13, 651-687. 